Hélcio Bezerra de Mello

\title{
Propostas de Roteamento para Redes Veiculares (VANETs) em Ambientes Urbanos
}

Tese apresentada ao Programa de Pós-graduação em Informática do Departamento de Informática da PUC-Rio como requisito parcial para obtenção Do título de Doutor em Informática

Orientador: Prof. Markus Endler 


\title{
Hélcio Bezerra de Mello
}

\section{Propostas de Roteamento para Redes Veiculares (VANETs) em Ambientes Urbanos}

Tese apresentada como requisito parcial para obtenção do grau de Doutor pelo Programa de Pós-graduação em Informática do Departamento de Informática do Centro Técnico Científico da PUC-Rio. Aprovada pela Comissão Examinadora abaixo assinada.

\author{
Prof. Markus Endler \\ Orientador \\ Departamento de Informática - PUC-Rio \\ Prof. Luiz Fernando Gomes Soares \\ Departamento de Informática - PUC-Rio
}

Prof. Marcus Vinícius Soledade Poggi de Aragão

Departamento de Informática — PUC-Rio

Prof. Jean-Marie Alexandre Farines Universidade Federal de Santa Catarina - UFSC

Prof. Luis Henrique Maciel Kosmalski Costa Universidade Federal do Rio de Janeiro - UFRJ

Prof. José Eugenio Leal

Coordenador Setorial do Centro Técnico Científico - PUC-Rio 
Todos os direitos reservados. É proibida a reprodução total ou parcial do trabalho sem autorização da universidade, do autor e do orientador.

\section{Hélcio Bezerra de Mello}

Graduou-se em Engenharia de Computação na Universidade Federal do Espírito Santo (UFES) em 2002. Obteve o título de Mestre em Informática na Pontifícia Universidade Católica do Rio de Janeiro (PUC-Rio) em 2004.

Ficha Catalográfica

Mello, Hélcio Bezerra de

Propostas de roteamento para redes veiculares (VANETs) em ambientes urbanos / Hélcio Bezerra de Mello ; orientador: Markus Endler. - 2009.

v., 125 f: il. ; $30 \mathrm{~cm}$

Tese (Doutorado em Informática)—Pontifícia Universidade Católica do Rio de Janeiro, Rio de Janeiro, 2009.

Inclui bibliografia.

1. Informática - Teses. 2. Roteamento. 3. Geocast. 4. VANETs. 5. Redes Veiculares. 6. Semáforos. 7. Grafo Multiplanar. I. Endler, Markus. II. Pontifícia Universidade Católica do Rio de Janeiro. Departamento de Informática. III. Título. 


\section{Agradecimentos}

Primeiramente a Deus, por tudo o que Ele já me concedeu e pelo que ainda me reserva.

À família, pelo amor e incentivo.

Ao $\mathrm{CNPq}$ e à PUC-Rio, pelo apoio financeiro aos meus estudos.

Ao meu orientador, prof. Markus Endler, pela paciência, compreensão e dedicação.

Aos funcionários do Departamento de Informática e das bibliotecas, por seus incansáveis esforços que tornaram a PUC-Rio a grande universidade que é hoje.

Aos colegas, pela amizade, companherismo e pelos momentos de alegria que me proporcionaram. 


\section{Resumo}

Mello, Hélcio Bezerra de; Endler, Markus. Propostas de Roteamento para Redes Veiculares (VANETs) em Ambientes Urbanos. Rio de Janeiro, 2009. 125p. Tese de Doutorado - Departamento de Informática, Pontifícia Universidade Católica do Rio de Janeiro.

Redes veiculares (VANETs - Vehicle Ad Hoc NETworks) constituem um caso especial de redes ad hoc em que os nós são veículos equipados com uma interface de comunicação sem fio. Esses veículos podem se mover a velocidades elevadas, e a transmissão de dados em cenários urbanos pode ser facilmente bloqueada por prédios ou outros obstáculos. Tais fatores contribuem para tornar a comunicação inter-veicular intermitente, e dificultar o roteamento de pacotes. Um dos principais desafios dos protocolos de roteamento em VANETs é evitar as ruas onde o volume de tráfego esteja baixo, uma vez que a escassez de veículos nessas ruas tende a impossibilitar a propagação de pacotes através delas. Por esse motivo, a informação sobre o volume de tráfego em cada rua é fundamental para se determinar a melhor rota entre dois veículos. Especificamente em cenários urbanos, a mudança de estado dos semáforos provoca uma flutuação do tráfego de veículos ao longo do tempo. Em vista disso, esta tese propõe o TLAR (Traffic Light Aided Routing), um novo algoritmo de roteamento para VANETs que explora a variação de estado dos semáforos para inferir quais ruas oferecerão uma maior probabilidade de sucesso de propagação de pacotes. Resultados de simulação mostram que o algoritmo apresenta um bom desempenho comparado ao de propostas existentes.

\section{Palavras-chave}

Roteamento. Geocast. VANETs. Redes Veiculares. Semáforos. Grafo Multiplanar. 


\section{Abstract}

Mello, Hélcio Bezerra de; Endler, Markus(advisor). Routing Proposals for Vehicular Networks (VANETs) in Urban Environments. Rio de Janeiro, 2009. 125p. DSc Thesis - Departamento de Informática, Pontifícia Universidade Católica do Rio de Janeiro.

VANETs (Vehicle Ad Hoc NETworks) are a special case of mobile ad hoc networks where vehicles are equiped with wireless communication interfaces. These vehicles may move at high speeds and data transmission in urban scenarios may easily be blocked by buildings and other sort of obstacles. Such factors contribute to make inter-vehicle communication intermitent and packet routing more difficult. One of the main challenges faced by routing protocols is avoiding low-traffic streets, where the lack of vehicles tend to make packet forwarding impossible. For this reason, traffic information on each street is essential for the computation of the best route between any given two vehicles. Specifically in urban scenarios, traffic light transitions cause significant fluctuations on traffic flow over time. Given this fact, this thesis proposes TLAR (Traffic Light Aided Routing), a new routing algorithm for VANETs that exploits traffic light transition timings in order to determine which streets will offer the greatest probabilities for successful packet forwarding. Simulation results indicate a good performance of this algorithm compared to existing approaches.

\section{Keywords}

Routing. Geocast. VANETs. Vehicular Networks. Traffic Lights. Multilayer Graphs. 


\section{Sumário}

1 Introdução $\quad 12$

1.1 Roteamento Geocast 14

$\begin{array}{lll}1.2 \text { Contribuições } & 17\end{array}$

2 Redes Veiculares $\quad 19$

2.1 Propagação de Radiofreqüência 20

$\begin{array}{lll}2.2 & \text { Aplicações } & 25\end{array}$

2.3 Aplicação de VANETs Considerada 29

3 Roteamento em VANETs 30

$\begin{array}{lll}3.1 & \text { SAR } & 30\end{array}$

3.2 GyTAR e A-STAR 31

$\begin{array}{lll}3.3 & \text { VADD } & 32\end{array}$

3.4 GeOpps 33

$\begin{array}{lll}3.5 & \text { GPSR } & 35\end{array}$

$\begin{array}{lll}3.6 & \text { STAR } & 37\end{array}$

$\begin{array}{lll}3.7 & \text { Resumo } & 40\end{array}$

4 Propostas de Algoritmos de Roteamento 42

4.1 Modelo de VANETs 42

4.2 TLAR 46

4.3 U-GPSR 64

$\begin{array}{lll}5 & \text { Experimentos } & \mathbf{6 7}\end{array}$

5.1 Ambiente de Simulação $\quad 67$

5.2 Experimentos 80

$\begin{array}{lll}5.3 & \text { Análise e Discussão } & 107\end{array}$

6 Conclusão 111

$\begin{array}{ll}\text { Referências Bibliográficas } & 113\end{array}$ 


\section{Lista de figuras}

2.1 Propagação no modelo two-ray ground. 21

2.2 Propagação de sinal por difração sobre prédios. 24

2.3 Exemplo do uso da técnica de raytracing. 24

2.4 Uso do radar no controle de cruzeiro adaptativo. 26

2.5 Controle de cruzeiro cooperativo agilizando a reação do veículo de trás.

2.6 Mesclagem de fluxos de veículos em uma auto-estrada. 27

3.1 Determinando o ponto mais próximo da rota de um veículo ao destinatário de um pacote.

3.2 Um exemplo de ótimo local.

3.3 Aplicação da regra da mão direita. 36

3.4 A regra da mão direita falhando em grafo não-planar. 36

3.5 Região que contém os vizinhos a serem eliminados. 37

4.1 Redução do tamanho dos obstáculos para fins de propagação. 44

4.2 (a) Exemplo de cenário para conversão em grafo. (b) Representação tradicional em grafo. (c) Grafo multiplanar correspondente 52

4.3 Uma situação onde $C C(X)$ é ajustada para $100 \%$. 54

4.4 Exemplo de ajuste para conectividades retas.

4.5 Selecionando o próximo hop de uma rota. 56

4.6 Exemplo de situação em que pode ocorrer a quebra da seqüência de super-vizinhos.

4.7 Prédios podem impedir a deteção de arestas que se cruzam. $\quad 64$

4.8 Exemplo de aplicação da estratégia de reparo do U-GPSR. $\quad 65$

5.1 Exemplo de arquivo de cruzamentos do SUMO. 68

5.2 Exemplo de arquivo de segmentos do SUMO. 68

5.3 Exemplo de topologia no SUMO. 69

5.4 Exemplo de descrição de fluxos de veículos. 69

5.5 Trecho de um relatório gerado por simulação do SUMO. 70

5.6 Exemplo de configuração de mobilidade no NS-2. 71

5.7 Procedimento adotado nas simulações. $\quad 72$

5.8 Rotas a serem seguidas por veículos injetados na topologia em $A . \quad 73$

5.9 Aglomeração de veículos próximos de um cruzamento. Veículos equipados estão indicados em vermelho.

5.10 Cálculo da largura da calçada para efeito de propagação.

5.11 Quantidade de pacotes entregues pelo TLAR em função de seus tempos de vida para alguns valores da constante de penalidade. A taxa de penetração é de $40 \%$, o intervalo de chegada de veículos é de 3 s e o alcance de comunicação é de $200 \mathrm{~m}$.

5.12 Quantidade de pacotes entregues pelo STAR em função de seus tempos de vida para alguns valores da constante SREG_HIGH. A taxa de penetração é de $40 \%$, o intervalo de chegada de veículos é de $3 \mathrm{~s}$ e o alcance de comunicação é de $200 \mathrm{~m}$. 
5.13 Quantidade de pacotes entregues pelo STAR em função de seus tempos de vida para alguns valores da constante SPROP_HIGH. A taxa de penetração é de $40 \%$, o intervalo de chegada de veículos é de $3 \mathrm{~s}$ e o alcance de comunicação é de $200 \mathrm{~m}$.

5.14 Quantidade de pacotes entregues pelo STAR em função de seus tempos de vida para alguns valores da constante TIMEOUT. A taxa de penetração é de $40 \%$, o intervalo de chegada de veículos é de $3 \mathrm{~s}$ e o alcance de comunicação é de $200 \mathrm{~m}$.

5.15 Cruzamento usado para análise das conectividades.

5.16 (a) Ângulo de visão dos veículos em um cruzamento. (b) Cálculo da visibilidade cruzada.

5.17 Efeito da variação dos parâmetros de simulação na conectividade cruzada: (a) Intervalo de chegada de veículos (b) Taxa de penetração (c) Alcance de comunicação.

5.18 Calculando a conectividade reta de $A B$.

5.19 Efeito da variação dos parâmetros de simulação na conectividade reta: (a) Intervalo de chegada de veículos (b) Taxa de penetração (c) Alcance de comunicação.

5.20 Quantidade de pacotes entregues em função de seus tempos de vida para algumas taxas de penetração. O intervalo de chegada de veículos é de 3s e o alcance de comunicação é de $200 \mathrm{~m}$.

5.21 Amostras de veículos equipados para algumas taxas de penetração (p). Veículos equipados são mostrados em vermelho. (a) $p=30 \%$. (b) $p=40 \%$. (c) $p=40 \%$ (superconjunto da amostra de (a)).

5.22 Quantidade de pacotes entregues em função de seus tempos de vida para alguns intervalos de chegada de veículos. A taxa de penetração é de $40 \%$ e o alcance de comunicação é de $200 \mathrm{~m}$.

5.23 Quantidade de pacotes entregues em função de seus tempos de vida para algumas margens de erro $\epsilon$. O intervalo de chegada de veículos é de 3s, a taxa de penetração é de $40 \%$ e o alcance de comunicação é de $200 \mathrm{~m}$.

5.24 Quantidade de pacotes entregues em função de seus tempos de vida para diferentes velocidades, acelerações e desacelerações. 0 intervalo de chegada de veículos é de $3 \mathrm{~s}$, a taxa de penetração é de $40 \%$ e o alcance de comunicação é de $200 \mathrm{~m}$.

5.25 Quantidade de pacotes entregues em função de seus tempos de vida para semáforos sincronizados (a) e dessincronizados (b e c). A defasagem dos semáforos é de no máximo $30 \mathrm{~s}$ (b) e $60 \mathrm{~s}$ (c). 0 intervalo de chegada de veículos é de $3 \mathrm{~s}$, a taxa de penetração é de $40 \%$ e o alcance de comunicação é de $200 \mathrm{~m}$.

5.26 Um cenário para a demonstração de uma possível melhoria para a estratégia de reparo do TLAR. 


\section{Lista de tabelas}

2.1 Parâmetros do modelo de shadowing calculados em uma área urbana. 23

3.1 Comparação dos protocolos de roteamento para VANETs.

4.1 Formato do cabeçalho de um pacote do TLAR.

5.1 Parâmetros das camadas física e MAC.

5.2 Desempenho dos algoritmos para taxa de penetração de 30\%, alcance de transmissão de $200 \mathrm{~m}$ e intervalo de chegada de veículos de 3 s.

5.3 Desempenho dos algoritmos para taxa de penetração de 40\%, alcance de transmissão de $200 \mathrm{~m}$ e intervalo de chegada de veículos de 3s.

5.4 Desempenho dos algoritmos para taxa de penetração de 50\%, alcance de transmissão de $200 \mathrm{~m}$ e intervalo de chegada de veículos de $3 s$.

5.5 Desempenho dos algoritmos para intervalo de chegada de veículos de 2 s, taxa de penetração de $40 \%$ e alcance de transmissão de 200 $\mathrm{m}$.

5.6 Desempenho dos algoritmos para intervalo de chegada de veículos de 3s, taxa de penetração de $40 \%$ e alcance de transmissão de 200 $\mathrm{m}$.

5.7 Desempenho dos algoritmos para intervalo de chegada de veículos de $4 \mathrm{~s}$, taxa de penetração de $40 \%$ e alcance de transmissão de 200 $\mathrm{m}$.

5.8 Taxas de chegada de veículos equipados nos experimentos.

5.9 Desempenho do TLAR para várias margens de erro nas conectividades. Intervalo de chegada de veículos: 3s, taxa de penetração: 40\%, alcance de transmissão: $200 \mathrm{~m}$.

5.10 Desempenho dos algoritmos para taxa de penetração de 40\%, alcance de transmissão de $200 \mathrm{~m}$ e intervalo de chegada de veículos de 3s. A velocidade máxima dos veículos pertence ao intervalo $[15,0 ; 20,0] \mathrm{m} / \mathrm{s}$, enquanto a aceleração e desaceleração se encontram, respectivamente, nos intervalos $[0,7 ; 0,9] \mathrm{m} / \mathrm{s}^{2}$ e $[4,0 ; 5,0] \mathrm{m} / \mathrm{s}^{2}$.

5.11 Desempenho dos algoritmos para taxa de penetração de 40\%, alcance de transmissão de $200 \mathrm{~m}$ e intervalo de chegada de veículos de 3s. A velocidade máxima dos veículos pertence ao intervalo $[15,0 ; 20,0] \mathrm{m} / \mathrm{s}$, enquanto a aceleração e desaceleração se encontram, respectivamente, nos intervalos $[0,5 ; 1,0] \mathrm{m} / \mathrm{s}^{2}$ e $[3,5 ; 5,5] \mathrm{m} / \mathrm{s}^{2}$.

5.12 Desempenho dos algoritmos para semáforos sincronizados, a uma taxa de penetração de 40\%, alcance de transmissão de $200 \mathrm{~m}$ e intervalo de chegada de veículos de $3 \mathrm{~s}$. 
5.13 Desempenho dos algoritmos para semáforos dessincronizados, a uma taxa de penetração de $40 \%$, alcance de transmissão de $200 \mathrm{~m}$ e intervalo de chegada de veículos de 3s. A defasagem máxima é de $30 \mathrm{~s}$.

5.14 Desempenho dos algoritmos para semáforos dessincronizados, a uma taxa de penetração de $40 \%$, alcance de transmissão de $200 \mathrm{~m}$ e intervalo de chegada de veículos de 3s. A defasagem máxima é de $60 \mathrm{~s}$. 